Article

\title{
Prediction of the Ideal Implant Size Using 3-Dimensional Healthy Breast Volume in Unilateral Direct-to-Implant Breast Reconstruction
}

\author{
Jeong-Hoon Kim $『$, Jin-Woo Park and Kyong-Je Woo * \\ Department of Plastic and Reconstructive Surgery, Ewha Womans University Mokdong Hospital, \\ College of Medicine, Ewha Womans University, 1071 Anyangcheon-ro, Yangcheon-gu, Seoul 07985, Korea; \\ kimsbrothers@hanmail.net (J.-H.K.); burnscar@naver.com (J.-W.P.) \\ * Correspondence: economywoo@gmail.com
}

Received: 21 August 2020; Accepted: 18 September 2020; Published: 24 September 2020

check for updates

\begin{abstract}
Background and objectives: There is no consensus regarding accurate methods for assessing the size of the implant required for achieving symmetry in direct-to-implant (DTI) breast reconstruction. The purpose of this study was to determine whether the ideal implant size could be estimated using 3D breast volume or mastectomy specimen weight, and to compare prediction performances between the two variables. Materials and Methods: Patients who underwent immediate DTI breast reconstruction from August 2017 to April 2020 were included in this study. Breast volumes were measured using 3D surface imaging preoperatively and at postoperative three months. Ideal implant size was calculated by correcting the used implant volume by the observed postoperative asymmetry in 3D surface imaging. Prediction models using mastectomy weight or 3D volume were made to predict the ideal implant volume. The prediction performance was compared between the models. Results: A total of 56 patients were included in the analysis. In correlation analysis, the volume of the implant used was significantly correlated with the mastectomy specimen weight $(\mathrm{R} 2=0.810)$ and the healthy breast volume $(\mathrm{R} 2=0.880)$. The mean ideal implant volume was $278 \pm 123 \mathrm{cc}$. The prediction model was developed using the healthy breast volume: Implant volume $(\mathrm{cc})=$ healthy breast volume $\times 0.78+26 \mathrm{cc}(\mathrm{R} 2=0.900)$. The prediction model for the ideal implant size using the 3D volume showed better prediction performance than that of using the mastectomy specimen weight ( $\mathrm{R} 2=0.900$ vs $0.759, p<0.001)$. Conclusions: The 3D volume of the healthy breast is a more reliable predictor than mastectomy specimen weight to estimate the ideal implant size. The estimation formula obtained in this study may assist in the selection of the ideal implant size in unilateral DTI breast reconstruction.
\end{abstract}

Keywords: 3D breast volume; direct-to-implant breast reconstruction; estimation of ideal implant size

\section{Introduction}

Nipple sparing mastectomy (NSM) and direct-to-implant (DTI) breast reconstruction have been gaining popularity because it is oncologically safe, requires less surgery and fewer visits, and is more cost-effective than two-stage expander/implant reconstruction [1-3]. Surgeons choose the implants considering the weight of the resected breast tissue, the patient anatomy as assessed by subjective linear measurements, the surgeon's experience, and the availability of implants. However, linear measurements including the height, width, and projection of the breasts are insufficient to describe the breast shape and size accurately, and small measurement discrepancies may lead to variations in volumetric implant size estimation and potentially unacceptable asymmetry. Although various methods have been developed, there is no universally accepted standard method for determining breast volume and for choosing the optimal implant size for breast reconstruction. 
In the last decade, advances in 3-dimensional surface imaging have produced techniques for handling vast data formats efficiently and generating precise 3-dimensional surface images [4-6]. The breast volume can be measured preoperatively using 3D surface imaging, and the 3D volumes are known to be significantly correlated with the mastectomy specimen weight [7]. Similar to the mastectomy specimen weight, the preoperative 3D volume of the breasts can assist the surgeon in calculating the ideal volume of the implant. However, there have been no guidelines developed regarding how to estimate ideal implant volume using 3D volume data of the breast. The purpose of this study was to determine whether the ideal implant size could be estimated using 3-dimensional breast volume or mastectomy specimen weight, and to compare prediction performances between these two variables.

\section{Materials and Methods}

\subsection{Study Population and Data Collection}

The study was conducted in accordance with the Declaration of Helsinki, and the protocol was approved by the institutional review board of Ewha Womans University Mokdong Hospital (No. 2020-07-046). Prospectively recorded data from consecutive patients who underwent immediate unilateral DTI reconstruction at a single institution between August 2017 and April 2020 were retrospectively reviewed. Patients who underwent bilateral breast reconstruction, simultaneous contralateral augmentation/reduction surgery, previous surgery on the affected breast side, and patients with incomplete records were excluded. Data on patient demographics, surgical procedures, mastectomy specimen weight, and implant size used were collected. Breast volumes were measured using 3D surface imaging preoperatively and at postoperative three months. The primary outcome was the ideal implant size, which was calculated by correcting the used implant volume by the observed postoperative asymmetry in 3D surface imaging. The independent variables were either the mastectomy specimen weight or the preoperative 3D volume of the healthy breast.

\subsection{Method for 3-Dimensional Surface Imaging and Volume Extraction}

Volumetric assessment of the breast was obtained using a Crisalix 3D ${ }^{\circledR}$ imaging scan (Crisalix, Lausanne, Switzerland). The Crisalix system is a cloud-based, 3-dimensional simulation program. 3D surface scanning was performed using a 3D sensor attached to a portable tablet to scan the patient's front and both sides in a standing position. The total time for each individual scan was a few seconds, and the total procedure time, including marking of breast landmarks, takes less than $10 \mathrm{~s}$. All 3D scans were performed with the patient in the same position. Patients were scanned in a standing position with the back of the feet and the shoulders touching the wall, and arms abducted with both wrists placed on the hips of the patients.

Data from the 3-dimensional scan are uploaded and merged to generate a 3-dimensional surface image, which is then rendered. This software program can then generate the curvature of the simulated chest wall and the indicated breast boundary from the torso curved to match the real body shape and thereby calculate the volume of the 3D breast image [8]. After completing the 3-dimensional surface image, the volume of the breast soft tissue was measured with consideration of $3 \mathrm{~mm}$ skin thickness (Figure 1). Postoperatively, the 3D surface imaging was repeated to compare the volumes of the reconstructed and healthy breasts at three months postoperative follow-up using the same protocol. 


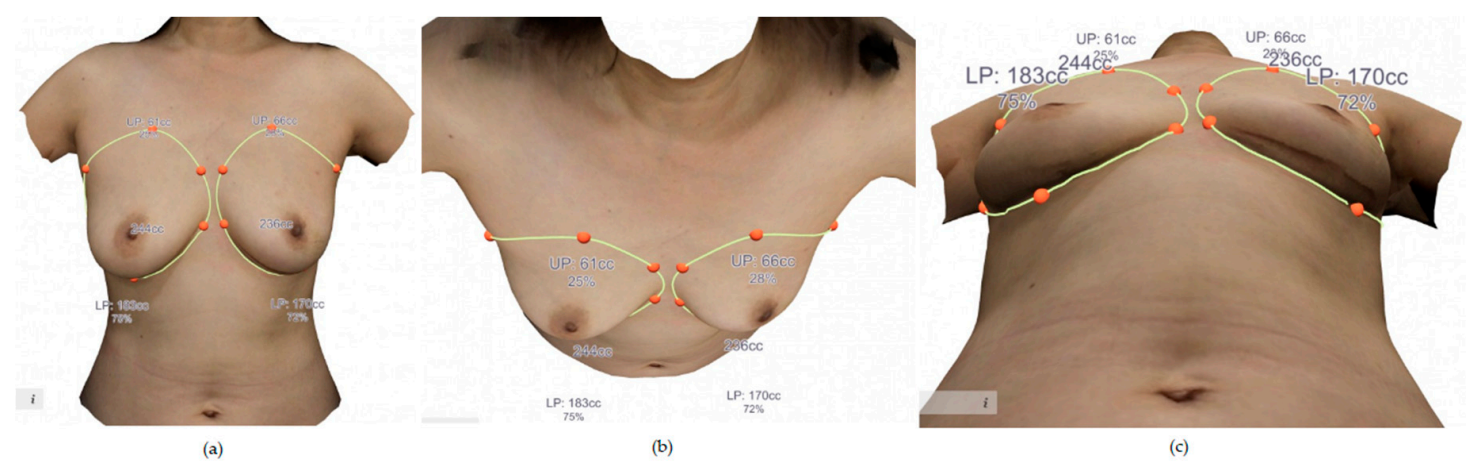

Figure 1. A case example of preoperative 3-D volumes of the breasts. (a) Anterior view. (b) Cephalic to caudal view. (c) Caudal to cephalic view.

\subsection{Reconstruction after Nipple-Sparing Mastectomy}

Oncologic surgeons performed the mastectomy, and the senior author (K.J.W.) performed all reconstructions. The implant size and type were determined by the surgeon considering the patient's breast dimensions, contralateral breast volume measured before surgery, and the resected mastectomy specimen weight to achieve symmetric breasts. The size of the implant used was determined after two or three temporary sizers were inserted and checking the breast symmetry by visual inspection and palpation. The implants were placed in the subpectoral or prepectoral spaces. In subpectoral DTI, an acellular dermal matrix (human cadaveric) of 6-8 $\times 16-18 \mathrm{~cm}$ size was used for inferior and lateral support and implant coverage. In prepectoral DTI, a $16 \times 16 \mathrm{~cm}$ or $18 \times 18 \mathrm{~cm}$ acellular dermal matrix was used to cover the implant.

\subsection{Calculation of Ideal Implant Size}

Because the implants used were not always the ideal size for symmetry in unilateral DTI breast reconstructions, an ideal implant size was calculated by correcting the used implant volume by the observed postoperative asymmetry in 3D surface imaging. The ideal implant volume $=$ Inserted implant volume $-\beta \times$ (Surgical side breast volume - Contralateral side breast volume measured postoperatively at three months after surgery). The $\beta$ was the slope of the linear regression model that was obtained using the preoperative breast volume as an independent variable and the inserted implant volume as the dependent variable. If the $\beta$ was 0.7, a $100 \mathrm{cc}$ increase of the breast volume resulted in a $70 \mathrm{cc}$ increase of the implant volume in the linear regression model.

\subsection{Statistical Analysis}

The mean with standard deviation or median with interquartile range were used to summarize continuous variables based on the distribution of the data. Pearson correlation coefficients between implant size and morphological factors, including mastectomy specimen weight and healthy breast volume, were first examined to determine the most suitable references for implant size choice. Linear regression analysis was performed to develop formulas predicting the optimal inserted implant volume.

After calculation of the ideal implant sizes, linear regression models were used to develop formulas to predict the ideal implant volume for symmetry using mastectomy specimen weight and preoperative healthy breast volume as predictor variables. The prediction performances using the two predictor variables were compared. Residual analysis was performed to assess the appropriateness of the linear regression model. The statistical significance was determined by $p<0.05$. All analyses were performed using SPSS version 23.0 (SPSS Inc., Chicago, IL, USA). 


\section{Results}

\subsection{Patient Demographics and Operative Data}

A total of 56 patients undergoing immediate unilateral DTI reconstruction were included in the analysis. The patients' mean age was $47.95 \pm 8.44$ years (IQR, 43.5-52.3) with a mean BMI of $22.77 \pm 2.50 \mathrm{~kg} / \mathrm{m}^{2}$ (IQR, 20.92-24.25). Nipple-sparing mastectomy was performed in $85.7 \%$ (48 of 56 patients), and skin-sparing mastectomy was performed in the remaining cases. Prepectoral placement of the implant was performed in $46.4 \%$, and subpectoral placement was performed in $53.6 \%$. The mean preoperative volume of the affected breast was $318 \pm 154 \mathrm{cc}(\mathrm{IQR}, 194-408)$ and that of the contralateral unaffected breast was $323 \pm 150 \mathrm{cc}(\mathrm{IQR}, 203-380)$. The mean mastectomy specimen weight was $287 \pm 128 \mathrm{~g}(\mathrm{IQR}, 181-348)$ and the mean volume of inserted implant was $288 \pm 107 \mathrm{cc}$ (IQR, 200-375) (Table 1).

Table 1. Clinical and surgical characteristics.

\begin{tabular}{|c|c|}
\hline No. of patients & 56 \\
\hline Age, mean $\pm S D$, yr & $47.95 \pm 8.44$ \\
\hline $\mathrm{BMI}$, mean $\pm \mathrm{SD}, \mathrm{kg} / \mathrm{m}^{2}$ & $22.77 \pm 2.50$ \\
\hline \multicolumn{2}{|l|}{ Cancer laterality } \\
\hline No. of right $(\%)$ & $35(62.5)$ \\
\hline No. of left $(\%)$ & $21(37.5)$ \\
\hline \multicolumn{2}{|l|}{ Mastectomy type } \\
\hline No. of nipple-sparing (\%) & $48(85.7)$ \\
\hline No. of skin-sparing (\%) & $8(14.3)$ \\
\hline Mastectomy specimen weight, mean $\pm \mathrm{SD}, \mathrm{g}$ & $287.6 \pm 128.2$ \\
\hline Inserted implant volume, mean $\pm \mathrm{SD}, \mathrm{cc}$ & $287.5 \pm 107.0$ \\
\hline Inserted $\mathrm{ADM}$ size, mean $\pm \mathrm{SD}, \mathrm{cm}^{2}$ & $204.0 \pm 82.9$ \\
\hline \multicolumn{2}{|l|}{ Preoperative volume of the breasts } \\
\hline Pre-operative volume of affected breast, mean $\pm \mathrm{SD}, \mathrm{cc}$ & $317.6 \pm 154.3$ \\
\hline Pre-operative volume of contralateral unaffected breast, mean $\pm S D, c c$ & $322.9 \pm 150.0$ \\
\hline \multicolumn{2}{|l|}{ Postoperative volume of the breasts } \\
\hline Post-operative volume of affected breast, mean $\pm \mathrm{SD}, \mathrm{cc}$ & $336.8 \pm 147.8$ \\
\hline Post-operative volume of contralateral unaffected breast, mean $\pm \mathrm{SD}, \mathrm{cc}$ & $321.2 \pm 161.1$ \\
\hline
\end{tabular}

In the analysis of preoperative 3D volume of the healthy and affected breasts, mean volume differences of the breasts were $49.5 \pm 39.8 \mathrm{cc}$ (IQR, 23.0-76.0 cc) (Figure 2). The mean percentage of volume differences was $15.8 \pm 13.4 \%$ (IQR, 8.5-23.1\%), and $32.1 \%$ of the patients (18 of 56) had over $20 \%$ volume differences between the healthy and affected breasts.

\subsection{Prediction Model for the Inserted Implant Volume}

The Pearson correlation coefficient of the mastectomy specimen weight was $0.900(p<0.001)$. In the linear regression analysis, a prediction model was developed (Figure 3).

1. Inserted implant volume $=0.75 \times$ mastectomy specimen weight $(\mathrm{g})+72 \mathrm{cc}(\mathrm{R} 2=81.0 \%, p<0.001)$.

The Pearson correlation coefficient of the healthy breast volume was $0.938(p<0.001)$. In the linear regression analysis, the inserted implant volume was better estimated with the model using healthy breast volume. 
2. Inserted implant volume $=0.66 \times$ healthy breast volume $+71 \mathrm{cc}(\mathrm{R} 2=88.0 \%, p<0.001)$.

The results of the residual analysis satisfied the assumptions of the linear regression models.

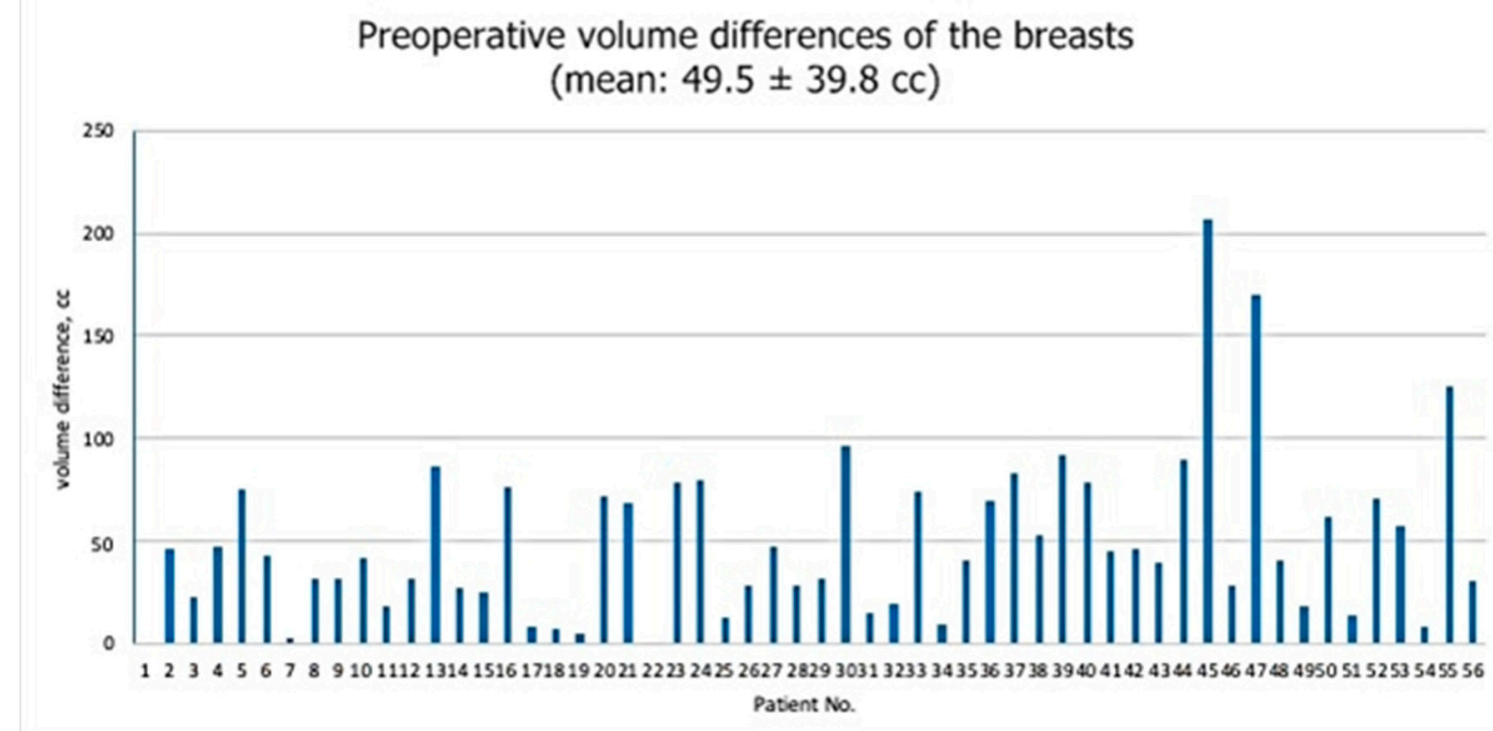

(a)

Percentage of preoperative volume differences of the breasts (mean: $15.8 \pm 13.4 \%$ )

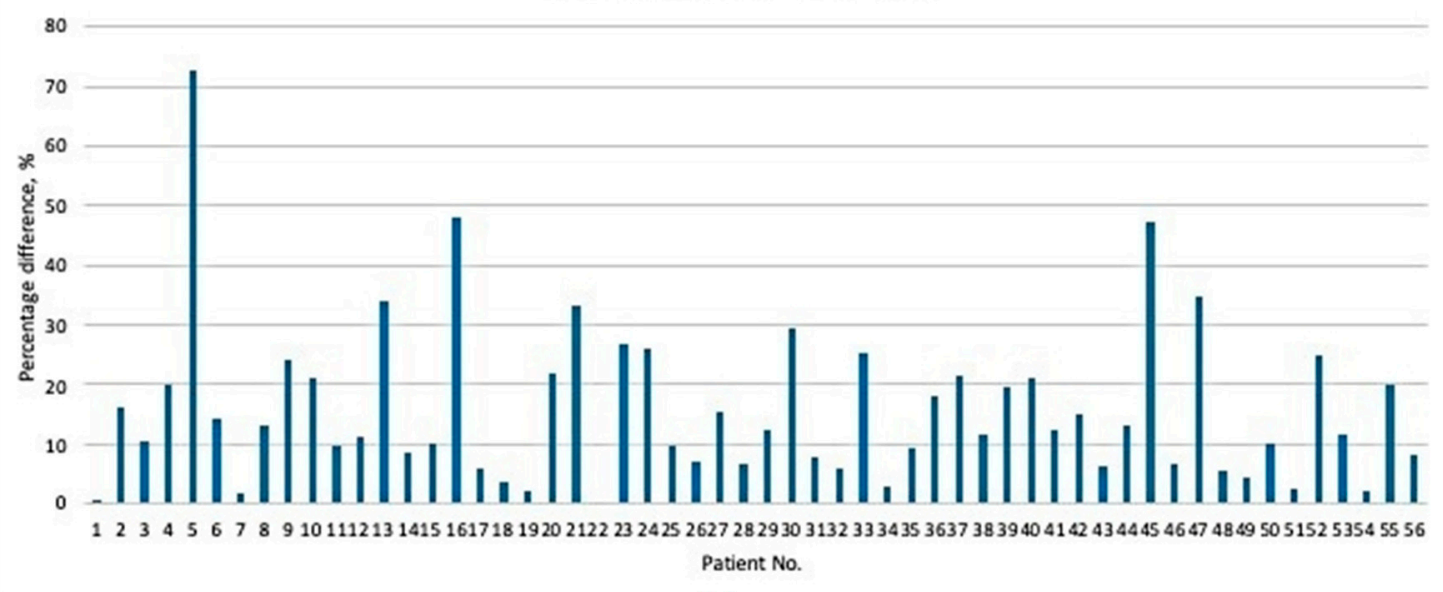

(b)

Figure 2. (a) Preoperative volume differences of the breasts. (b) Percentage of preoperative volume differences of the breasts. The percentage of volume differences were calculated by (1-reconstruction side breast volume/healthy breast volume) $\times 100$. 

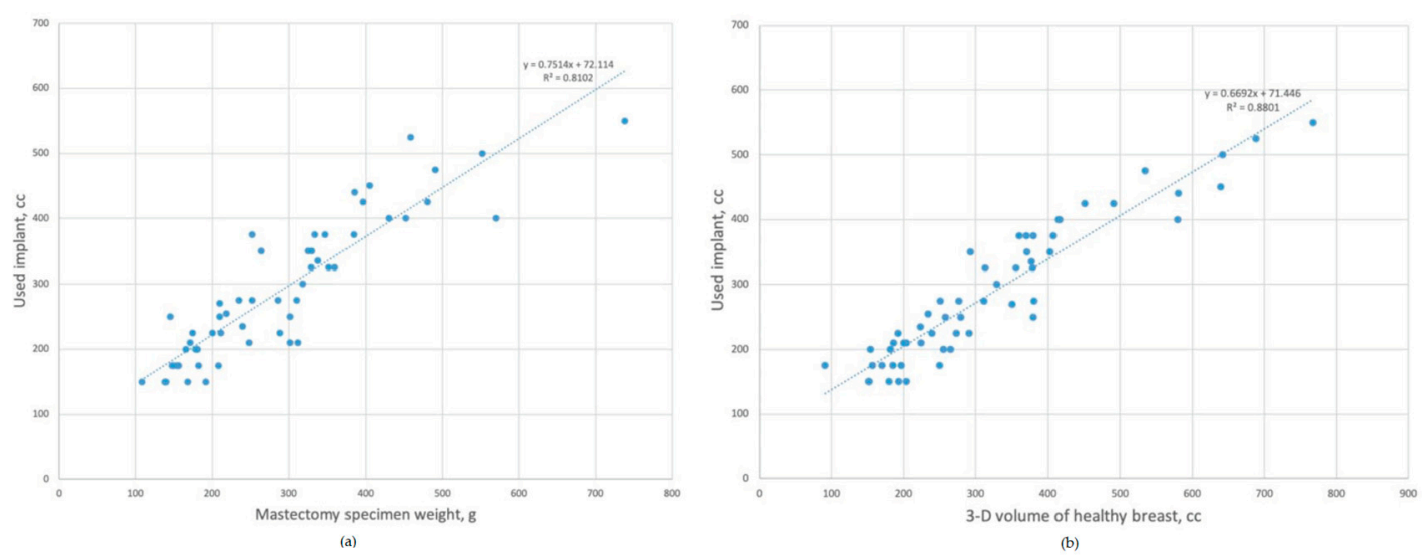

Figure 3. Predication model for inserted implant. (a) Scatterplot and the linear regression model using mastectomy specimen weight as a predictor variable. (b) Scatterplot and the linear regression model using 3-D volume of healthy breast as a predictor variable.

\subsection{Prediction Model for Ideal Implant Volume}

Because the inserted implant volume could not be considered as an ideal implant volume for symmetry, ideal implant volume was calculated by comparing the postoperative volumes of both breasts. The mean volume of the ideal implant size was $278 \pm 123 \mathrm{cc}$ (IQR, 181-338 cc). The prediction model of an ideal implant volume was as follows (Figure 4).

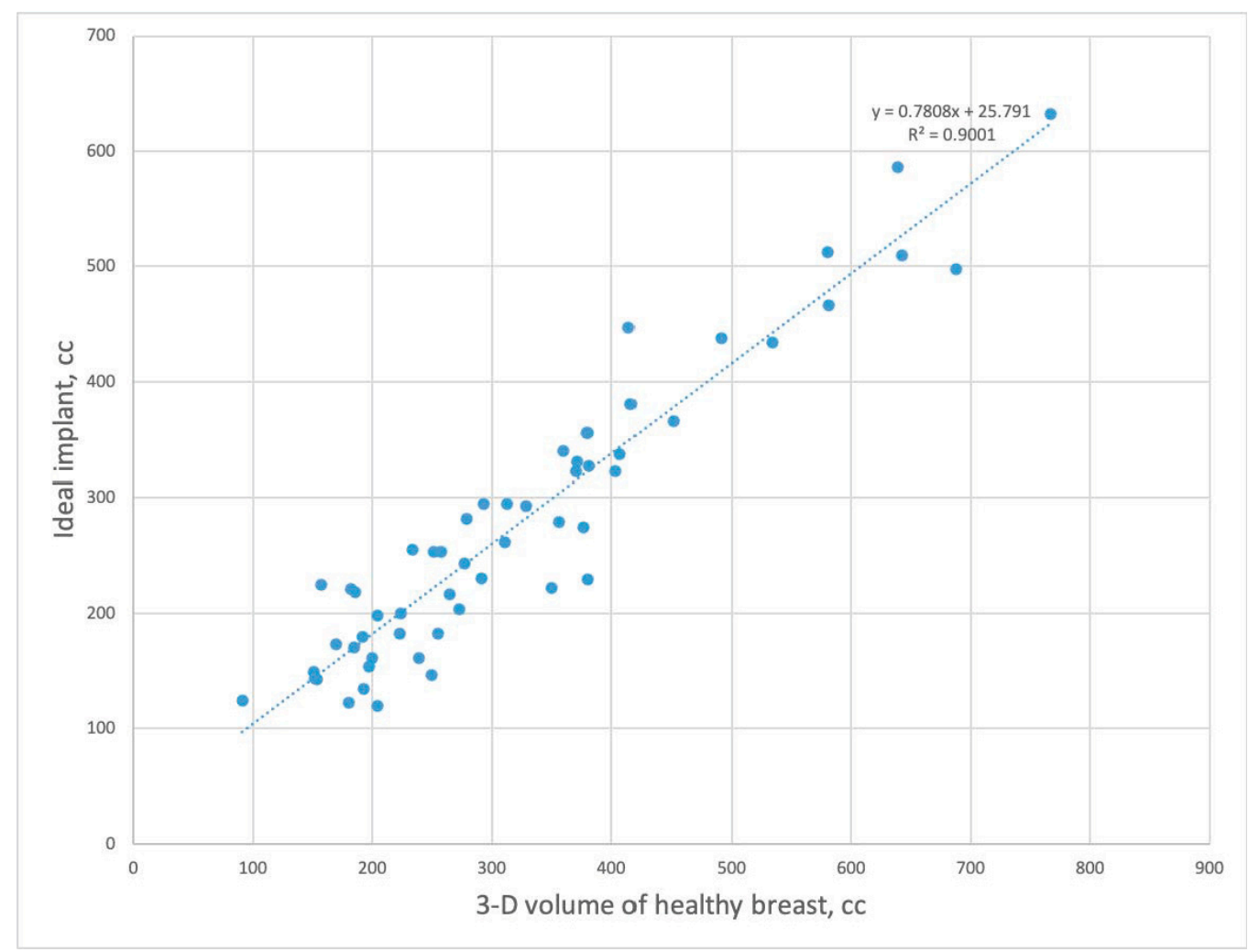

Figure 4. Predication model for ideal implant. Scatterplot and the linear regression model using 3-D volume of healthy breast as a predictor variable.

1. Mastectomy specimen weight as a predictor variable.

Ideal implant volume $=$ mastectomy specimen weight $\times 0.84+37 \mathrm{cc}(\mathrm{R} 2=75.9 \%, p<0.001)$. 
2. Healthy breast volume as a predictor variable.

Ideal implant volume $=$ healthy breast volume $\times 0.78+25 \mathrm{cc}(\mathrm{R} 2=90.0 \%, p<0.001)$.

The results of the linear regression models showed that an ideal implant volume could be predicted by both healthy breast volume and mastectomy specimen weight. In terms of prediction performance, the ideal implant volume could be better estimated using the 3D volume of the healthy breast compared to the mastectomy specimen weight (coefficient of determination $=90.0 \% \mathrm{vs} .75 .9 \%$ ). The results of the residual analysis satisfied the assumptions of linear regression models.

\section{Discussion}

The selection of an ideal size of implant is crucial for achieving symmetry in immediate DTI breast reconstruction. The current study demonstrated that the ideal implant size could be estimated by a linear regression model using either mastectomy specimen weight $(p<0.001)$ or the 3D volume of the healthy breast $(p<0.001)$. Moreover, we found that the 3D volume could predict the ideal implant size more accurately than the mastectomy specimen weight $(\mathrm{R} 2=0.900 \mathrm{vs}$. 0.759$)$.

Many surgeons subjectively evaluate the breast volumes and symmetry. However, subjective measurement of breast volume cannot be reliable and is not accurate [9]. Mastectomy specimen weight has been proposed to be used as a reference for the selection of ideal implant size [10-13]. The density of mastectomy specimen weight is known to be $1.06 \mathrm{~g} / \mathrm{mL}$ [14]. However, the density of the breast is different among patients because the proportions of fibro-glandular tissue and fat tissue volume of the breast are different. The tumor itself can also change the density and weight of the affected breast.

Furthermore, innate breast asymmetry cannot be taken into account when using the mastectomy specimen as a reference to estimate the ideal implant size. Recently, Liu et al. found that the incidence of significant asymmetry of the breast mound was 94 percent [15]. We also found that most patients had innate asymmetry of their breast volumes. The mean percentage of volume differences was $15.8 \pm 13.4 \%$ (IQR, 8.5-23.1\%), and about one-third of the patients (18 of 56) had over $20 \%$ volume differences between the healthy and affected breasts in our study. Georgiou et al. reported that the implant size could be predicted by the mastectomy specimen weight using a linear regression model, but there were limitations since the coefficient of determination $(R 2)$ was less that $0.5(R=0.66)[11]$.

2-dimensional images of CT or MRI and subsequent 3D reconstruction can be used for breast volume measurement [12,16-20]. However, 3D reconstruction of the 2D images are complex and need additional software. Moreover, it is not completely objective because boundary annotation of the breast tissue has to be performed manually or threshold of breast tissue has to be arbitrarily determined on the $2 \mathrm{D}$ images.

Recently, 3D surface imaging has gained acceptance in clinical use in breast surgery [4-6]. 3D surface imaging has been used for the selection of implant size and for creating simulations for augmentation mammoplasty [21-24]. Yip et al. reported that breast volume could be measured by 3D surface imaging (Pearson's correlation, $\mathrm{R}=0.95, p<0.001$ ) [7]. Roostaeian et al. evaluated the accuracy of the 3D surface imaging and reported that preoperative simulation by 3D surface imaging can predict postoperative breast volume with more than $90 \%$ accuracy [25]. Previous studies have suggested the potential use of 3D surface imaging for measuring the breast volume for selection of the ideal implant size in DTI breast reconstructions. We found that the 3D volume of the healthy breast measured by 3D surface imaging could be used for estimation of the ideal implant size. We also demonstrated that the $3 \mathrm{D}$ volume of the healthy breast showed better prediction performance than the mastectomy specimen weight (coefficient of determination, $R 2=0.900$ vs. 0.759). Implant size can be easily calculated by multiplying the healthy breast volume by 0.78 and adding $25 \mathrm{cc}$ (ideal implant volume = healthy breast volume $\times 0.78+25 \mathrm{cc}$ ).

In order to develop a predictive model for satisfactory results after surgery, it is desirable to set the dependent variable as an ideal implant size rather than the inserted implant size. Most of the previous studies developed a prediction model based on the inserted implant. However, the inserted implant was not validated as an ideal implant size because postoperative evaluation was not performed in 
the previous studies. In the present study, we calculated the ideal implant size by correcting the inserted implant volume using the observed postoperative asymmetry in postoperative 3D surface imaging. Pohlmann et al. also calculated the ideal implant size by simply adding or subtracting the postoperative volume differences of both breasts [26]. However, $100 \mathrm{cc}$ differences of the breast volume do not correspond to $100 \mathrm{cc}$ differences of an implant. In the analysis of our data using inserted implant size and 3D breast volume, beta was $0.66(\mathrm{R} 2=88.0 \%, p<0.001)$. The results suggested that a $100 \mathrm{cc}$ difference corresponded to $66 \mathrm{cc}$ of implant. Therefore, multiplying beta by the postoperative volume difference would be better for calculation of the ideal implant size.

One of significant findings of our study was that the beta values of the prediction model using $3 \mathrm{D}$ breast volume (0.78) and mastectomy specimen weight (0.84) were both less than 1 . This result suggests that the ideal implant size should be smaller than the 3D breast volume or mastectomy specimen weight except when the breast size is small ( $<200 \mathrm{cc}$ ) (Figure 5). According to our prediction model, difference between ideal implant volume and 3-D volume of the healthy breast (or mastectomy weight) becomes larger as the breast size increases. This would be true because extent of oncologic resection is usually larger than the dimensions of the implant. In this case, the inserted implant makes central portions of the breast mound without covering peripheral area of the mastectomy defect. Similar with our study, Back et al. reported that long-term patient satisfaction was highest in a patient group whose implant volume to mastectomy specimen weight was $71.9 \%$ [10].
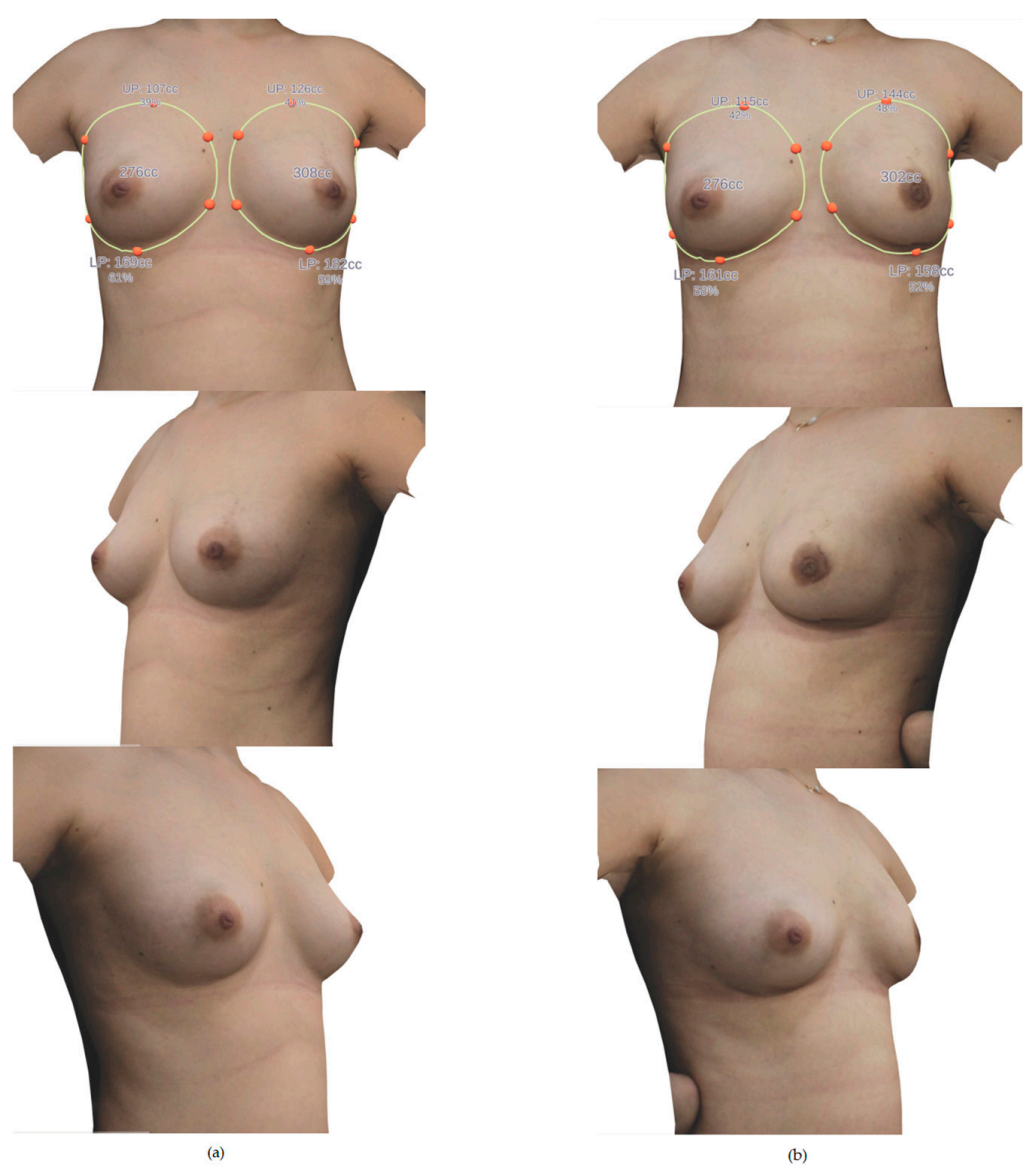

Figure 5. A case example of pre and postoperative 3-D volumes of the breasts. (a) Preoperative 3-D image. A 41-year-old woman with a diagnosis of left breast cancer. The volume of both breasts was $276 \mathrm{cc}$ and $308 \mathrm{cc}$. The resected mastectomy specimen weight was $252 \mathrm{~g}$ and the inserted implant size was 275 cc. The operation was performed through periareolar incision, and the implant was inserted into the subpectoral plane. (b) Postoperative 3-D image. The volume of both breasts after three months of surgery was $276 \mathrm{cc}$ and $302 \mathrm{cc}$. The volume on the reconstruction side was $9.4 \%$ larger. 
Considering that our retrospective data set was relatively small, and the developed formulas have not been fully tested, the generalizability of these two formulas may requires more testing, especially in different populations. However, a relatively high coefficient of determination $(\mathrm{R} 2=0.90)$ could be obtained with statistical significance. The coefficient of determination in our prediction model $(\mathrm{R} 2=0.90)$ was higher than that of previous studies $[11,13,26]$. In terms of selection of the ideal implant in unilateral DTI breast reconstruction, implant volume is not the only factor to be considered for breast symmetry. Other various factors including breast width, breast height, projection, upper pole fullness, and degree of ptosis need to be considered together for the ideal implant volume for symmetry. Additionally, the size of the acellular dermal matrix influences the final volume of the reconstructed breast. The thickness and dimensions of the acellular dermal matrix should be considered, especially in prepectoral DTI, in which the implant is covered 360 degrees with the acellular dermal matrix.

\section{Conclusions}

Healthy breast volume measured by 3D surface imaging is a more accurate predictor than mastectomy specimen weight to estimate the implant volume for symmetry in DTI breast reconstruction. The estimation formula obtained in this study may assist in the selection of ideal implant size in unilateral DTI breast reconstruction.

Author Contributions: Conceptualization, K.-J.W.; Data curation, J.-H.K.; Formal analysis, J.-H.K. and J.-W.P.; Investigation, J.-W.P.; Methodology, J.-W.P. and K.-J.W.; Supervision, K.-J.W.; Writing-original draft, J.-H.K.; Writing-review and editing, J.-W.P. and K.-J.W. All authors have read and agreed to the published version of the manuscript.

Funding: This research received no external funding.

Conflicts of Interest: The authors declare no conflict of interest.

\section{References}

1. Azouz, V.; Lopez, S.; Wagner, D.S. Surgeon-Controlled Comparison of Direct-to-Implant and 2-Stage Tissue Expander-Implant Immediate Breast Reconstruction Outcomes. Ann. Plast. Surg. 2018, 80, $212-216$. [CrossRef] [PubMed]

2. Kamali, P.; Koolen, P.G.L.; Ibrahim, A.M.S.; Paul, M.; Dikmans, R.E.; Schermerhorn, M.L.; Lee, B.T.; Lin, S.J. Analyzing Regional Differences over a 15-Year Trend of One-Stage versus Two-Stage Breast Reconstruction in 941,191 Postmastectomy Patients. Plast. Reconstr. Surg. 2016, 138, 1e-14e. [CrossRef] [PubMed]

3. Serrurier, L.C.J.; Rayne, S.; Venter, M.; Benn, C.-A. Direct-to-Implant Breast Reconstruction without the Use of an Acellular Dermal Matrix Is Cost Effective and Oncologically Safe. Plast. Reconstr. Surg. 2017, 139, $809-817$. [CrossRef] [PubMed]

4. Chang, J.B.; Small, K.H.; Choi, M.; Karp, N.S. Three-Dimensional surface imaging in plastic surgery: Foundation, practical applications, and beyond. Plast. Reconstr. Surg. 2015, 135, 1295-1304. [CrossRef]

5. Galdino, G.M.; Nahabedian, M.; Chiaramonte, M.; Geng, J.Z.; Klatsky, S.; Manson, P. Clinical Applications of Three-Dimensional Photography in Breast Surgery. Plast. Reconstr. Surg. 2002, 110, 58-70. [CrossRef]

6. Tzou, C.H.; Artner, N.M.; Pona, I.; Hold, A.; Placheta, E.; Kropatsch, W.G.; Frey, M. Comparison of three-dimensional surface-imaging systems. J. Plast. Reconstr. Aesthet. Surg. 2014, 67, 489-497. [CrossRef]

7. Yip, J.M.; Mouratova, N.; Jeffery, R.M.; Veitch, D.E.; Woodman, R.J.; Dean, N.R. Accurate assessment of breast volume: A study comparing the volumetric gold standard (direct water displacement measurement of mastectomy specimen) with a 3D laser scanning technique. Ann. Plast. Surg. 2012, 68, 135-141. [CrossRef]

8. Georgii, J.; Eder, M.; Bürger, K.; Klotz, S.; Ferstl, F.; Kovács, L.; Westermann, R. A Computational Tool for Preoperative Breast Augmentation Planning in Aesthetic Plastic Surgery. IEEE J. Biomed. Health Inform. 2014, 18, 907-919. [CrossRef]

9. Henseler, H.; Hille-Betz, U.; Vogt, P. Validation of Subjective Estimates of Female Breast Volume and Comparison with Objective Methods. Handchir. Mikrochir. Plast. Chir. 2015, 47, 371-377. [CrossRef]

10. Baek, W.Y.; Byun, I.H.; Kim, Y.S.; Lew, D.H.; Jeong, J.; Roh, T.S. Patient Satisfaction with Implant Based Breast Reconstruction Associated with Implant Volume and Mastectomy Specimen Weight Ratio. J. Breast Cancer 2017, 20, 98-103. [CrossRef] 
11. Georgiou, C.; Ihrai, T.; Chamorey, E.; Flipo, B.; Chignon-Sicard, B. A formula for implant volume choice in breast reconstruction after nipple sparing mastectomy. Breast 2012, 21, 781-782. [CrossRef]

12. Shia, W.; Yang, H.-J.; Wu, H.-K.; Lin, S.-L.; Lai, H.-W.; Huang, Y.-L.; Chen, D.-R. Implant volume estimation in direct-to-implant breast reconstruction after nipple-sparing mastectomy. J. Surg. Res. 2018, 231, 290-296. [CrossRef] [PubMed]

13. Wazir, U.; Chehade, H.E.H.; Choy, C.; Kasem, A.; Mokbel, K. A Study of the Relation Between Mastectomy Specimen Weight and Volume with Implant Size in Oncoplastic Reconstruction. Vivo 2019, 33, 125-132. [CrossRef] [PubMed]

14. Parmar, C.; West, M.; Pathak, S.; Nelson, J.; Martin, L. Weight versus volume in breast surgery: An observational study. JRSM Short Rep. 2011, 2, 1-5. [CrossRef] [PubMed]

15. Liu, C.; Luan, J.; Mu, L.; Ji, K. The Role of Three-Dimensional Scanning Technique in Evaluation of Breast Asymmetry in Breast Augmentation: A 100-Case Study. Plast. Reconstr. Surg. 2010, 126, 2125-2132. [CrossRef]

16. Chae, M.P.; Hunter-Smith, D.J.; Spychal, R.T.; Rozen, W.M. 3D volumetric analysis for planning breast reconstructive surgery. Breast Cancer Res. Treat. 2014, 146, 457-460. [CrossRef]

17. Erić, M.; Anderla, A.; Stefanović, D.; Drapšin, M. Breast volume estimation from systematic series of CT scans using the Cavalieri principle and 3D reconstruction. Int. J. Surg. 2014, 12, 912-917. [CrossRef]

18. Fujii, T.; Yamaguchi, S.; Yajima, R.; Tsutsumi, S.; Asao, T.; Kuwano, H. Accurate Assessment of Breast Volume by Computed Tomography Using Three-dimensional Imaging Device. Am. Surg. 2012, 78, 933-935. [CrossRef]

19. Kim, H.; Mun, G.-H.; Wiraatmadja, E.S.; Lim, S.-Y.; Pyon, J.-K.; Oh, K.S.; Lee, J.E.; Nam, S.J.; Lim, S.Y. Preoperative Magnetic Resonance Imaging-Based Breast Volumetry for Immediate Breast Reconstruction. Aesthet. Plast. Surg. 2015, 39, 369-376. [CrossRef]

20. Yoo, A.; Minn, K.W.; Jin, U.S. Magnetic Resonance Imaging-Based Volumetric Analysis and Its Relationship to Actual Breast Weight. Arch. Plast. Surg. 2013, 40, 203-208. [CrossRef]

21. Creasman, C.N.; Mordaunt, D.; Liolios, T.; Chiu, C.; Gabriel, A.; Maxwell, G.P. Four-Dimensional Breast Imaging, Part II: Clinical Implementation and Validation of a Computer Imaging System for Breast Augmentation Planning. Aesthet. Surg. J. 2011, 31, 925-938. [CrossRef] [PubMed]

22. Donfrancesco, A.; Montemurro, P.; Hedén, P. Three-Dimensional simulated images in breast augmentation surgery: An investigation of patients' satisfaction and the correlation between prediction and actual outcome. Plast. Reconstr. Surg. 2013, 132, 810-822. [CrossRef] [PubMed]

23. Gladilin, E.; Gabrielova, B.; Montemurro, P.; Hedén, P. Customized Planning of Augmentation Mammaplasty with Silicon Implants Using Three-Dimensional Optical Body Scans and Biomechanical Modeling of Soft Tissue Outcome. Aesthet. Plast. Surg. 2010, 35, 494-501. [CrossRef] [PubMed]

24. Vorstenbosch, J.; Islur, A. Correlation of Prediction and Actual Outcome of Three-Dimensional Simulation in Breast Augmentation Using a Cloud-Based Program. Aesthet. Plast. Surg. 2017, 133, 481-490. [CrossRef]

25. Roostaeian, J.; Adams, W.P., Jr. Three-Dimensional Imaging for Breast Augmentation: Is This Technology Providing Accurate Simulations? Aesthet. Surg. J. 2014, 34, 857-875. [CrossRef]

26. Pöhlmann, S.T.L.; Harkness, E.; Taylor, C.J.; Gandhi, A.; Astley, S.M. Preoperative implant selection for unilateral breast reconstruction using 3D imaging with the Microsoft Kinect sensor. J. Plast. Reconstr. Aesthet. Surg. 2017, 70, 1059-1067. [CrossRef]

(C) 2020 by the authors. Licensee MDPI, Basel, Switzerland. This article is an open access article distributed under the terms and conditions of the Creative Commons Attribution (CC BY) license (http://creativecommons.org/licenses/by/4.0/). 